\title{
L'immunité humorale intestinale
}

L'IgA sécrétoire se lie aux antigènes dans la lumière intestinale. Elle joue ainsi un rôle fondamental dans la protection contre des antigènes d'origine alimentaire comme les bactéries, les toxines, les virus, les parasites et certaines protéines.

Jean Claude Rambaud Professeur à la faculté de médecine Lariboisière-Saint-Louis, Université Paris VII.

Chef de service à l'hôpital SaintLazare.

Marc Halphen

Chef de clinique à l'hôpital SaintLazare.

Marc Lemaire

Interne du Chu de Poitiers.

\section{REFERENCES}

I. Besredka A. La vaccination contre les états typhoides par la voie buccale. Ann Inst Pasteur 1919; $33: 882-903$.

2. Davies A. An investigation into the serological properties of dysentery stools. Lancet 1922; ii : I OO9-I 2.

3. Heremans JF, Heremans MT, Schultze ME. Isolation and description of a few properties of $\beta_{2}$ A-globulin of human serum. Clin Chim Acta 1959; $4: 96-102$

4. Tomasi TB, Tan EM, Solomon A, Prendergast RA. Characteristics of an immune system common to certain external secretions. 7 Exp Med 1965; I 21 : IOI-24.

5. Bienenstock J, Befus AD. Mucosal immunology. Immunology 1980; 4 I : 249-70.

\section{ADRESSE}

Hôpital Saint-Lazare, I07, rue du Faubourg Saint-Denis, 750 ro Paris.

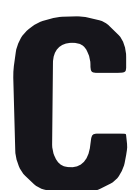
'est dans le premier quart de ce siècle que Besredka, à l'Institut Pasteur, démontra la possibilité d'immuniser des lapins, puis des ctres humains contre Salmonella parathyphi $B$ par administration orale de la bactérie inactivée [1]. A la même époque, Davies [2] montrait l'apparition précoce d'anticorps spécifiques dans les fèces de malades atteints de dysenterie bacillaire, avant que ne s'élève le taux des agglutinines sériques. Mais ce n'est qu'à partir de 1959 que ces données totalement oubliées trouvèrent leur explication dans la découverte de l'immunoglobuline $\mathrm{A}$ (IgA) par Heremans et ses collaborateurs [3]. La prédominance de cette nouvelle classe d'immunoglobulines dans le lait, dans le suc intestinal et les autres sécrétions exocrines de tous les mammifères [4] fut dès lors très rapidement mise en évidence, ainsi que la structure dimérique de cette $\operatorname{Ig}$ A sécrétoire (IgAs) et la présence en son sein du composant sécrétoire (CS) et de la chaîne polypeptidique J; sans oublier la compréhension du caractère essentiellement local de sa synthèse et des mécanismes de son transport transépithélial [5]. C'est enfin depuis les années 70 qu'ont été précisées l'origine et la circulation des cellules lymphoïdes B impliquées dans cette synthèse, ainsi que l'intégration de ce tissu lymphoide asso- cié au tractus digestif ou GALT (tableau I) dans un ensemble plus vaste comprenant la totalité des muqueuses en contact avec le milieu extérieur ou MALT [5].

\section{Structure et fonctions de I'lgA sécrétoire}

L'IgAs est faite d'un dimère d'IgA $7 \mathrm{~S}$, où les deux molécules d'immunoglobuline sont unies par la chaîne de jonction $\mathrm{J}$, et associées au CS ( figure I). Il existe deux sous-classes d'Ig A, l'Ig $\mathrm{A}_{2}$ se distinguant notamment de l'IgA par l'absence de ponts disulfure entre chaînes lourdes et chaînes légères. La chaîne J est une glycoprotéine de 15000 daltons, d'origine plasmocytaire et unie à la partie C-terminal des chaînes $\alpha$ par des liaisons covalentes. Présente dès les premiers stades du développement de la lignée lymphoïde $B$, la chaîne $J$ est indispensable à la polymérisation intracytoplasmique de l'IgA monomérique, mais n'en est pas la seule responsable. Elle est aussi nécessaire aux liaisons, covalente et non covalente, de l'IgA au CS. Celui-ci est un polypeptide de 65000 daltons, provenant d'un précurseur dit CS transmembranaire synthétisé par les entérocytes [6]. Outre son rôle dans le transport transépithélial de l'IgA, qui sera envisagé plus loin, il protège l'IgAs de la protéolyse dans la lumière intestinale. Il y a également 


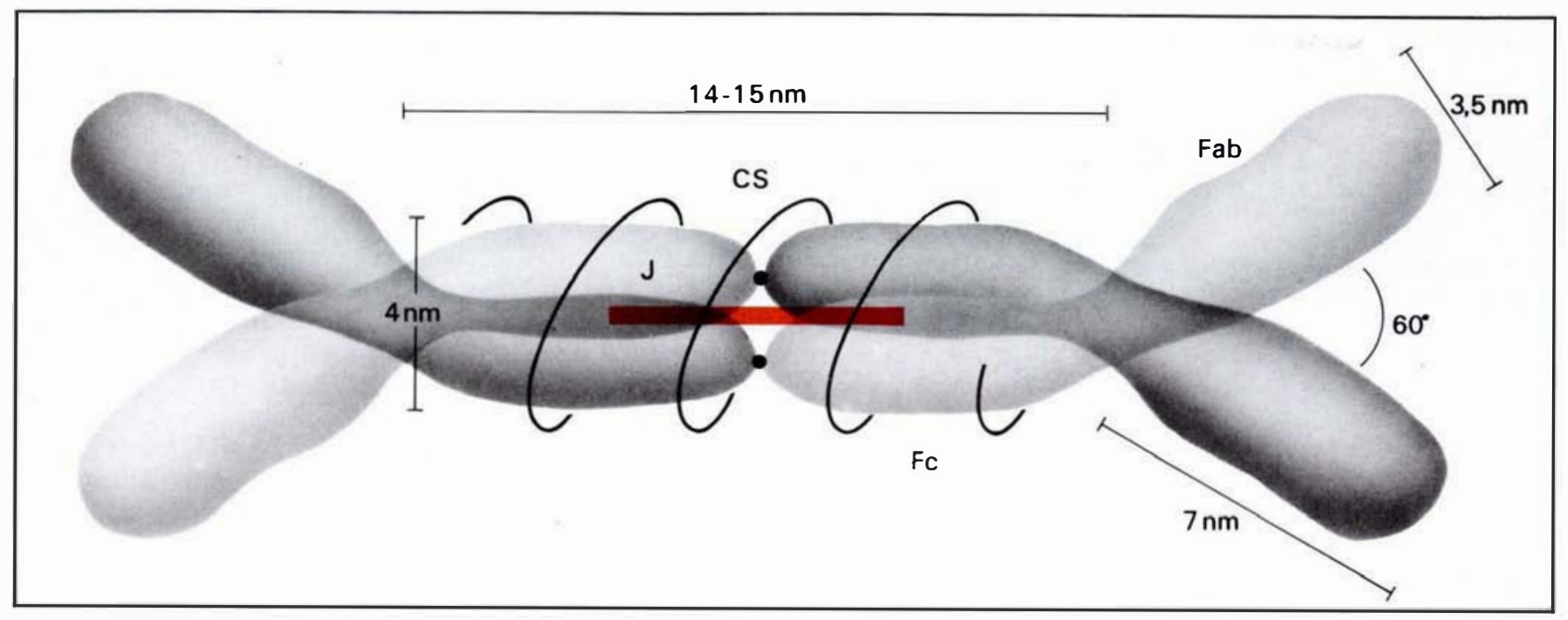

dans l'intestin de petites quantités d'IgM, elle aussi présente sous forme sécrétoire (IgMs). L'IgMs est un pentamère d'IgM monomère dans lequel la présence de la chaîne $\mathrm{J}$ permet une liaison non covalente au CS.

Le principal mécanisme d'action de l'IgAs est, grâce à la présence de quatre sites anticorps par molécule, l'agglutination et l'enveloppement (coaling) des antigènes intra-luminaux [5]. Elle n'active pas le complément, du moins par la voie classique, et n'est donc pas en principe bactéricide. Cependant, l'existence d'un pouvoir lytique par activation de la voie alterne du complément et en présence de lysozyme a été démontrée in vitro. Toujours in vitro, la fixation de l'IgAs sur le récepteur à l'IgA $7 \mathrm{~S}$ des monocytes, granulocytes et lymphocytes a pu être mise en évidence, ainsi que l'existence d'une cytotoxicité cellulaire vis-à-vis de bactéries telles que Shigella Flexneri et Salmonella typhimurium, dépendante d'anticorps spécifiques de classe IgAs [7]. L'inhibition par l'IgAs de l'adhérence des germes à la muqueuse, facteur essentiel de leur virulence, joue un rôle important dans la protection de l'intestin contre les bactéries pathogènes, telles que Vibrio cholerae et Escherichia coli entérotoxinogène [8]. Cette inhibition peut être obtenue par le développement d'anticorps dirigés spécifiquement contre les pili (ou fimbriae) de ces agents
Figure I. Représentation schématique de la structure de l'lgA sécrétoire du colostrum. Chaque molécule d'IgA sécrétoire est formée de deux molécules d'IgA monomérique de constante de sédimentation $7 S$. Elles sont reliées entre elles, au niveau de leur fragment constant $F c$, par la chaîne de jonction \}, d'origine plasmocytaire, et par le composant sécrétoire $C S$, d'origine entérocytaire. Les fragments Fab portent les quatre sites anticorps de l'immunoglobuline. D'après $/ 3^{\prime} /$.

Tableau I

RÉPARTITION DES POPULATIONS LYMPHOÏDES DU TISSU LYMPHOÏDE ASSOCIÉ AU TRACTUS DIGESTIF (GALT) HUMAIN

Formations lymphoïdes agrégées

- plaques de Peyer

- formations lympho-épithéliales de l'appendice et de l'anneau de Waldeyer

- follicules lymphoïdes isolés disséminés

- ganglions mésentériques.

Population lymphoïde disseminée

- Iymphocytes intra-épithéliaux

- plasmocytes de la lamina propria

- lymphocytes de la lamina propria. 


\section{RÉFÉRENCES}

6. Solari R, Krachenbuhl JP. The biosynthesis of secretory component and its role in the transepithclial transport of IgA dimer. Immunol Today r $985 ; 6: 17-20$.

7. Tagliabue A, Nencioni I, Villa I, Keren I)F Lowell GH, Boraschi 1). Antibody-dependent cell mediated anti bacterial activity of intestina lymphocytes with sccretory-IgA. Nature 1983; $306: 184-6$

8. Svanborg-Eden C., Andersson B, Hagberg I, et al. Receptor analogues and anti-pili antibodics as inhibitors of bactcrial attachment in vivo and in vitro. Ann NY.Acad Sici 1983; 409 : 580-92.

9. Porter P, Linggood M A. Novel mucosal antimicrobial functions interfering with the plasmidmediated virulence determinants of adherence and drug-resistance. Ann $N Y^{\circ}$.Acad Sci 1983; $409: 564-79$

ı. Ogra PL, Karzon DT. Distribution of Polio virus antibody in serum nasopharynx and alimentary tract following segmental immunization of lower alimentary tract with Polio vaccine. 7 Immunol 1969; $102: 1423-31$.

I I. Jonard PP, Rambaud JC, 1)ive C, Vacrman JP, Galian A, I)elacroix DL. Secretion of immunoglobulins and plasma proteins from the jejunal mucosa: transport rate and origin of polymeric Ig A. 7 Clin Intest $1984 ; 74: 525-35$

12. Mostor $\mathrm{KE}$, Friedlander $\mathrm{M}$, Blobel $\mathrm{G}$. 'The receptor for transepithelial transport of $\operatorname{Ig} \mathrm{A}$ and IgM contains multiple immunoglobulin like domains. Nature I 984; 308: 37-43.

13. Brandtzaeg P, Baklien K. Immunoglobulinproducing cells in the intestine in health and discasc. Clin Gastroenterol 1976; 5: 251-69.

I4. Owen RI, Jones AI.. F,pithelial cell specialization within human Peycr's patches: an ultrastructural study of intestinal lymphoid follicles. Gastroenterology' 1974; $66: 189-203$.

15. Wilders MMI, I)rexhale HA, Weltewreden E.F, et al. Viciled intestinal mononuclear cells: Part of the antigen presenting system. Immunology' I $983 ; 4^{8}: 453-60$

16. Butcher F.C; Stevens SK, Reichert RA, Scollay RG, Weissman II.. Lymphocyte endothelial cell recognition in lymphocyte migration and the segregation of mucosal and non mucosal immunity. In: Strober W, Hanson LA, Scll KW, cds. Recent adzances in mucosal immunity. New York: Raven Press, 1982 : 3-23.

17. Kawanishi H, Saltzman LE, Strober W Mechanisms regulating Ig A class specific immunoglobulin production in murine gut associated lymphoid tissues: T cells derived from Pcycr's patches that switch sIgM B cells to sIgA B cells in vitro. 7 Exp Med $1983 ; 175: 433-50$.

I8. Kiyono H, McGhec JR, Mosteller I.M, el al. Murine Pcycrs' patch T cell clones characterization of antigen specific helper $\mathrm{T}$ cells for immunoglobulin A responses. 7 Exp Med 1982; 156 : I I I 5-30. pathogènes, ce qui permet l'élaboration de vaccins oraux plus efficaces. L'IgAs peut, en outre, induire la perte de plasmides codant pour des facteurs de colonisation des pili, tel l'antigène K $88 \quad$ d'E. Coli $0149 \mathrm{~K} 91$ [9]. Enfin, l'abondance de l'IgAs au sein du mucus couvrant l'épithélium intestinal lui permet d'engluer les bactéries dans celui-ci et de rendre impossible leur adhérence au glycocalyx entérocytaire. La capacité d'inhiber l'adhérence bactérienne se double d'une activité neutralisante vis-à-vis des toxines de poids moléculaire élevé comme le choléragène et la toxine thermolabile d'E. Coli En revanche, les toxines de faible poids moléculaire, comme la toxine thermostable d'E. Coli, ne sont pas antigéniques. Par son activité agglutinante, l'IgAs est aussi capable d'inhiber in vitro la réplication des poliovirus et de prévenir in vivo la colonisation virale dans les segments du tractus gastrointestinal préalablement immunisés $[10]$. Une activité semblable a été démontrée contre des virus responsables de gastroentérites infantiles, tels que les rotavirus et certains adénovirus. Des anticorps agglutinants de classe IgAs dirigés contre des protéines alimentaires ont été détectés dans la sécrétion intestinale. Ils pourraient ainsi, mais ceci reste à démontrer formellement, "exclure " ces antigènes et, en limitant leur absorption sous forme non digérée - absorption faible mais réelle, même à l'âge adulte - réduire leur immunigénicité systémique.

Le taux des Ig As dirigées contre les antigènes parasitaires suit la cinétique de l'infestation. Cependant, certains parasites, tels Giardia Lamblia, persistent dans l'intestin malgré la sécrétion d'anticorps IgAs spécifiques. L'IgE fixée à la surface des mastocytes de la muqueuse intestinale joue par contre un rôle majeur dans l'éradication de certains parasites, tel Nippostrongylus brasiliensis chez le rongeur, mais cette immunoglobuline n'est pas d'origine locale [11]. I a possibilité pour certains parasites de devenir résidents par modification de leur antigénicité, et de prévenir ainsi l'infestation par des souches plus virulentes, est partagée par les germes de la flore bac- térienne saprophyte de l'intestin distal. D'une part, en effet, la colonisation du tractus digestif par les micro-organismes vivants joue un rôle essentiel dans le développement normal du GALT, comme cela a été démontré chez l'animal axénique et chez le nouveau-né [5]. D'autre part, malgré cette capacité de stimuler le système immunitaire intestinal et, notamment, de provoquer la sécrétion d'anticorps spécifiques IgAs, la flore saprophyte est parfaitement tolérée par l'hôte et reste très stable dans le temps. Il est probable que, sous la pression immunologique du GAI.T, les bactéries ayant colonisé l'intestin doivent, sous peine d'être éliminées, se modifier en variants génétiques moins immunogènes.

\section{Origine et transport}

Dans un perfusat de $40 \mathrm{~cm}$ du jéjunum proximal humain, le débit d'IgAs est en moyenne de $312 \mathrm{mg}$ par $24 \mathrm{~h}$, alors que celui de l'IgG, principale classe d'immunoglobulines sériques, n'est que de $50 \mathrm{mg}$ [11]. De plus, le pourcentage d'IgA $_{2}$ par rapport à I'IgA totale est, comme pour toutes les sécrétions exocrines, plus élevé que dans le sérum (tableau II). Ces données impliquent que l'IgAs de l'intestin est produite localement et/ou transportée à partir du plasma par un mécanisme sélectif. De fait, les études immunohistochimiques des nombreux plasmocytes de la lamina propria jéjunale ont montré que $80 \%$ de ceux-ci synthétisaient de I'IgA (avec des proportions d'Ig $\mathrm{A}_{1}$ et $\mathrm{d}^{\prime} \mathrm{Ig} \mathrm{A}_{2}$ très voisines de celles retrouvées pour l'IgAs intra-luminale), $17 \%$ de l'IgM et $3 \%$ seulement de l'IgG (tableau III). Quatre vingt dix pour cent des éléments de cette population plasmocytaire synthétisent la chaîne J. Dans la moëlle osseuse, seulement vingt pour cent des plasmocytes synthétisent cette chaîne.

I.e transport vers la lumière intestinale de $\operatorname{l}$ 'IgA dimérique ainsi synthétisée et sécrétée dans le milieu interstitiel de la lamina propria s'effectue par un mécanisme actif transentérocytaire où le CS joue un rôle majeur. Initialement identifié comme le polypeptide qui, avec l'IgA 
dimérique, forme l'IgAs, il est en fait synthétisé par l'entérocyte sous une forme dite transmembranaire [12]. D'un poids moléculaire de 95 ooo daltons, cette glycoprotéine se localise au niveau de la membrane latéro-basale de l'entérocyte, où elle est formée d'une séquence ectoplasmique de cinq domaines homologues un à un, d'une séquence intramembranaire ainsi que d'une séquence endoplasmique. Il existe un certain degré d'homologie entre les régions variables de l'IgA et les domaines ectoplasmiques du CS. Cette similitude entre récepteur et ligand a déjà été décrite dans d'autres systèmes, tel que celui de la cobalamine et de ses récepteurs. Elle permet d'inclure le CS dans le groupe des molécules qui, impliquées dans la réponse immunitaire, présentent des séquences homologues de celles des immunoglobulines (antigènes du système majeur d'histocompatibilité, récepteur cellulaire $\mathrm{T}$ ) et dérivent probablement d'un gène ancestral commun [12]. Une fois formés, les complexes IgA dimérique-CS sont endocytés. Au pôle apical de la cellule épithéliale, le contenu vésiculaire est relargué dans la lumière intestinale, sans que soit connu le moment où le $\mathrm{CS}$ passe de sa forme transmembranaire à sa forme sécrétoire [6]. Le CS est synthétisé même en l'absence de plasmocytes dans la lamina propria, comme dans l'hypogammaglobulinémie commune variable, et la présence constante d'une fraction libre, dans le liquide, de perfusat intestinal [11] montre que, chez le sujet normal, il n'est pas l'étape limitante de la sécrétion intra-luminale de l'IgAs [6]. Il n'en est plus nécessairement de même lorsqu'il existe une hyperplasie des plasmocytes synthétisant de l'IgA dimérique dans la paroi intestinale, comme dans un cas personnel récent. Il s'ensuit alors un passage passif d'Ig A dimérique dans la lumière digestive, et surtout dans le sang circulant. Chaque molécule de CS subit la conversion de la forme transmembranaire à la forme sécrétoire. Le CS se comporte donc comme un "récepteur sacrifié " ne pouvant servir qu'une fois. L'utilisation de traceurs marqués à permis de montrer que, chez l'homme, $2 \%$

\begin{tabular}{|c|c|c|}
\hline \multicolumn{3}{|c|}{$\begin{array}{c}\text { Tableau II } \\
\text { POURCENTAGES D'IgA POLYMĖRE (IgAp) ET D'IgA } 2 \\
\text { DANS LE SERUM ET LES SĖCRÉTIONS EXOCRINES DE L'HOMME (a) }\end{array}$} \\
\hline origine $\lg A$ & $\lg A p / \lg A(b)$ & $\lg A_{2} / \lg A(b)$ \\
\hline serum \% & 18 & $19 \pm 6$ \\
\hline salive $\%$ & 96 & $37 \pm 13$ \\
\hline sécrétion intestinale \% & 95 & $30 \pm 5$ \\
\hline lait \% & 96 & $35 \pm 3$ \\
\hline larmes \% & 95 & $41 \pm 5$ \\
\hline sécrétion bronchique $\%$ & 82 & $33 \pm 9$ \\
\hline bile $\%$ & 65 & $26 \pm 9$ \\
\hline
\end{tabular}

(a) D'après $|32|$

(b) Résultats donnés en pourcentage de l'IgA totale, \pm déviation standard.

\begin{tabular}{|c|c|c|c|c|}
\hline \multicolumn{5}{|c|}{$\begin{array}{c}\text { Tableau III } \\
\text { DENSITÉ ET RÉPARTITION DES PLASMOCYTES } \\
\text { DANS LA MUQUEUSE INTESTINALE (a) }\end{array}$} \\
\hline & \multirow{2}{*}{$\begin{array}{c}\text { Nombre total } \\
\text { de plasmocytes (c) }\end{array}$} & \multicolumn{3}{|c|}{$\begin{array}{c}\text { répartition } \\
\text { des plasmocytes (\%) }\end{array}$} \\
\hline & & $\lg A$ & $\lg M$ & $\lg G$ \\
\hline jéjunum & $128 \pm 38$ & $\begin{array}{c}\lg A=80 \pm 7 \\
\text { (b) }\left\{\begin{array}{l}\lg A_{1}=64,9 \\
\lg A_{2}=35,1\end{array}\right.\end{array}$ & $17 \pm 6$ & $2,6 \pm 1,6$ \\
\hline iléon & $45 \pm 15$ & $83 \pm 5$ & $11 \pm 4$ & $5 \pm 2,2$ \\
\hline colon-rectum & $143 \pm 58$ & $90 \pm 4$ & $6 \pm 3,5$ & \\
\hline
\end{tabular}

(a) D'après $|13|$.

(b) D'après $|I I|$.

(c) Résultat donné par unité de muqueuse $(500 \mu \mathrm{m}) \pm$ déviation standard.

de l'IgAs sécrétée par le jéjunum provenaient de l'IgA dimérique sérique, qui, comme les molécules synthétisées localement, se lie au CS [11]. L'Ig As salivaire et, si elle existe, l'IgAs gastrique sont rapidement détruites par la pepsine en milieu acide, et ne contribuent donc pas significativement au pool intraintestinal de l'IgAs. La contribution du pancréas exocrine est certainement faible; celle de la bile peut être évaluée à $5 \%$, alors qu'elle est majeure chez le rongeur.

Du fait de son haut poids moléculaire limitant son extravasation vasculaire, presque toute l'IgMs sécrétée dans la lumière jéjunale est synthétisée localement $[11,13]$ et est transportée selon les mêmes modalités que l'IgAs. En cas de déficit sélectif en IgA, l'accroissement de la synthèse locale d'IgM fait que la concentration globale d'immunoglo- 
bulines sécrétoires dans l'intestin diffère peu de la normale. Cependant, cette "compensation" de l'IgAs par l'IgMs ne semble pas parfaite, comme en témoignent la présence fréquente d'anticorps sériques de classe $\operatorname{IgG}$ anti-protéines alimentaires, ainsi que la possibilité d'une colonisation bactérienne du grêle proximal, responsable d'une malabsorption sévère. La concentration dans un perfusat jéjunal des immunoglobulines autres que l'IgAs et l'IgMs est, soit exclusivement le reflet d'un transport passif à partir du plasma (IgG, IgE), soit pour partie la conséquence d'une synthèse locale (IgA monomère) [11]. Toutes ces protéines sont extrudées lors de la desquamation entérocytaire à l'apex des villosités intestinales.

Si la structure, les fonctions et la sécrétion de l'IgAs intestinale sont acuellement assez bien connues, en revanche les modalités de la présentation des antigènes intra-luminaux aux cellules immunocompétentes ainsi que l'acquisition de l'isotype Ig A d'une part, l'origine et la circulation des cellules B du GALT d'autre part, sont encore en partie du domaine de l'hypothèse. Néanmoins, les travaux expérimentaux entrepris depuis une vingtaine d'années ont fourni quelques bases solides à l'édifice.

$\mathrm{La}$ présentation des antigènes intra-luminaux aux cellules immunocompétentes a lieu essentiellement dans les plaques de Peyer et dans les follicules lymphoïdes isolés.

\section{Acquisition de l'isotype IgA}

Il apparaît cependant possible que cette présentation ait lieu tout au long de l'intestin, dont les cellules épithéliales expriment un antigène Ia-like (molécules exprimées sur la membrane des cellules impliquées dans la reconnaissance des antigènes et sous la dépendance du locus HLA-DR). La structure des plaques de Peyer est schématisée dans la figure 2. A leur niveau, les villosi-
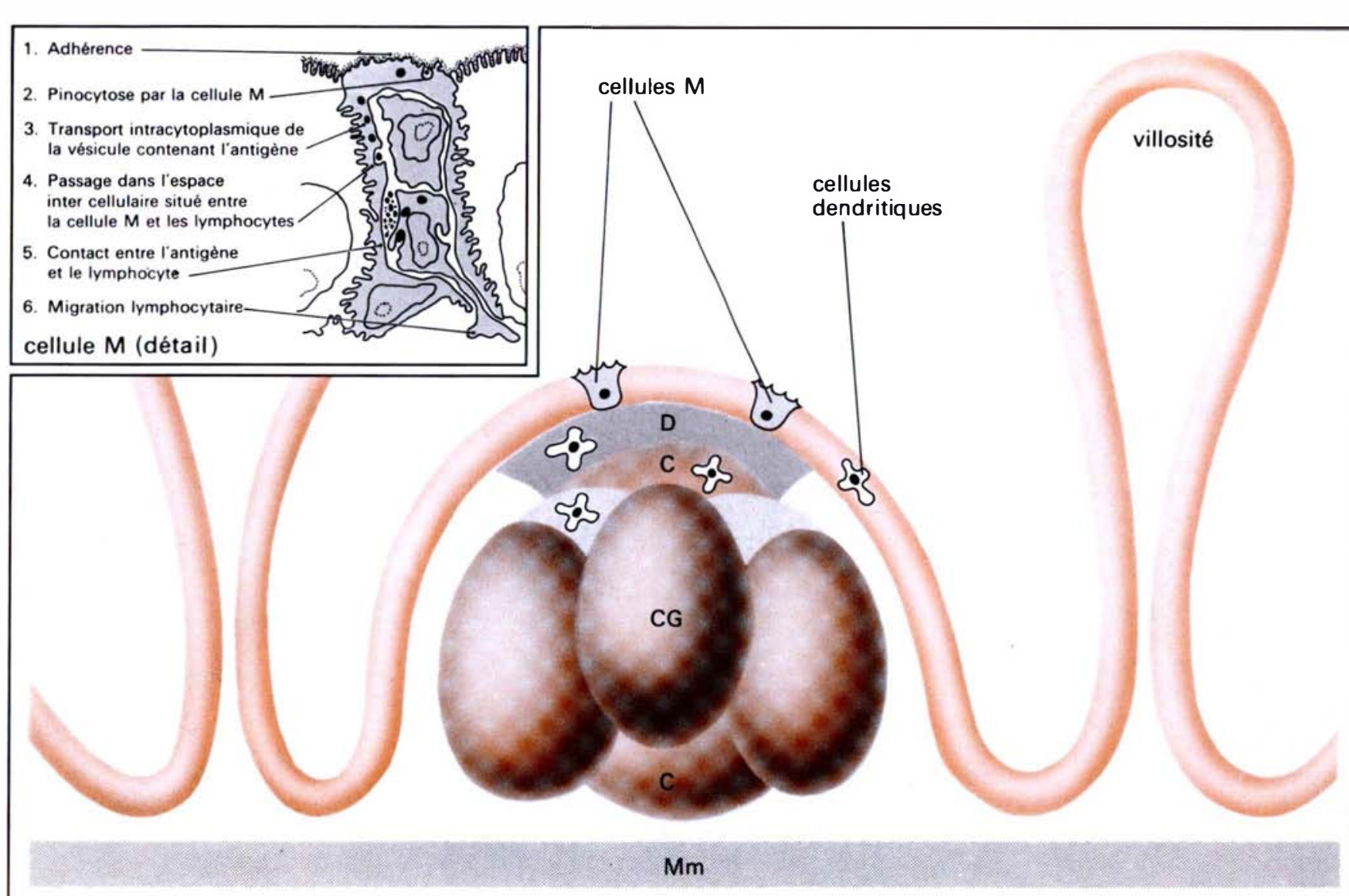

$\mathrm{Mm}$

Figure 2. Représentation schématique de la plaque de Peyer. Chaque plaque de Peyer est faite de la réunion de plusieurs follicules lymphoides, comportant un centre germinatif $(C G)$ entouré d'une couronne de petits lymphocytes $(C)$, et surmontés d'une zone mixte, $B$ et $T$ dépendante, le dôme $(D)$. Les villosités intestinales s'interrompent au niveau des plaques de Peyer, dont l'épithélium comporte des cellules $M$ (Schéma de la cellule $M$ d'après /33/) spécialisées dans la capture, le transport et la présentation des antigènes aux lymphocytes contenus dans le "trou" cytoplasmique de la cellule. Les follicules lymphoides comportent principalement des cellules lymphoïdes $B$ (zones gris-rose), les zones interfolliculaires des cellules $T$ (zones pointillées gris). Les cellules dendritiques seraient des macrophages intervenant dans la présentation des antigènes aux lymphocytes. 
tés s'interrompent et font place à un épithélium comportant des cellules spécialisées dites cellules $M$ [14]. Celles-ci comportent des microvillosités courtes et espacées, et sont pourvues de longues expansions cytoplasmiques enveloppant des lymphocytes $B$ et $T$ du dôme surmontant les follicules lymphoïdes. Leur rôle est d'absorber par pinocytose les antigènes intra-luminaux et de les transporter par un système de canaux tubulo-vésiculaires. Elles peuvent peut-être présenter directement les antigènes aux lymphocytes, mais cette fonction macrophagique semble en fait l'attribut des cellules dendritiques, avec lesquelles elles sont en contact étroit [1 5]. Les lymphocytes ainsi stimulés subissent une transformation blastique et une expansion clonale.

Contrairement à l'hypothèse ancienne disant que les plaques de Peyer sont l'équivalent chez les mammifères de la bourse de Fabricius des oiseaux, celles-ci sont des structures lymphoïdes périphériques. Chez l'adulte, elles reçoivent leur population lymphoïde $B$ de la moelle osseuse, par extravasation au niveau des veinules post-capillaires [16]. L'acquisition de l'isotype $\operatorname{Ig} \mathrm{A}$ par les cellules blastiques qui en dérivent, a fait l'objet de nombreuses études permises par l'isolement des lymphocytes $\mathrm{T}$ des plaques de Peyer de la souris. Dans un premier système, des clones $\mathrm{T}$ se sont avérés capables d'induire la commutation de classe $\operatorname{Ig} \mathrm{M} \rightarrow \operatorname{Ig} \mathrm{A}$, mais ne permettaient ni la prolifération, ni la maturation cellulaires, qui étaient dues à l'activité d'une autre cellule $\mathrm{T}$ auxilliaire (Th) [17]. Dans un autre système, les clones T obtenus induisaient non seulement une réponse de type $\operatorname{IgA}$, mais aussi de type IgG et IgM. La multiplication des cellules blastiques d'isotype $\operatorname{Ig} \mathrm{A}$ était due à la coopération d'une cellule Th spécifique appelée Th alpha, et d'une autre cellule Th spécifique de l'antigène et $\mathrm{H}_{2}$ (système majeur d'histocompatibilité de la souris) restreinte, mais sans spécificité isotypique [18]. Dans un tel système, la commutation de classe
$\operatorname{IgM} \rightarrow \operatorname{Ig} A$ serait un événement très précoce, génétiquement préprogrammé, qui ne serait déclenché ni par l'antigène, ni par les cellules $\mathrm{T}$, mais par le micro-environnement, et notamment les lipopolysaccharides des entérobactéries [19] ( figure 3). Cette hypothèse pourrait finalement rendre compte de la possibilité de réponses anticorps de type $\operatorname{Ig} A s$ contre des antigènes thymo-dépendants chez la souris nude athymique.

Quel qu'en soit le mécanisme inducteur et régulateur, la réponse locale de type IgAs à une stimulation antigénique peut être associée à une suppression de la réponse systémique secondaire de type IgM et IgG, même si l'antigène est réadministré par voie parentérale. Le mécanisme de cette tolérance systémique est complexe. La formation de complexes solubles circulants antigène-IgA, éliminés par voie biliaire, a été incriminée. L'immunisation par voie orale pourrait également conduire à la production de cellules $\mathrm{T}$ suppressives-cytotoxiques

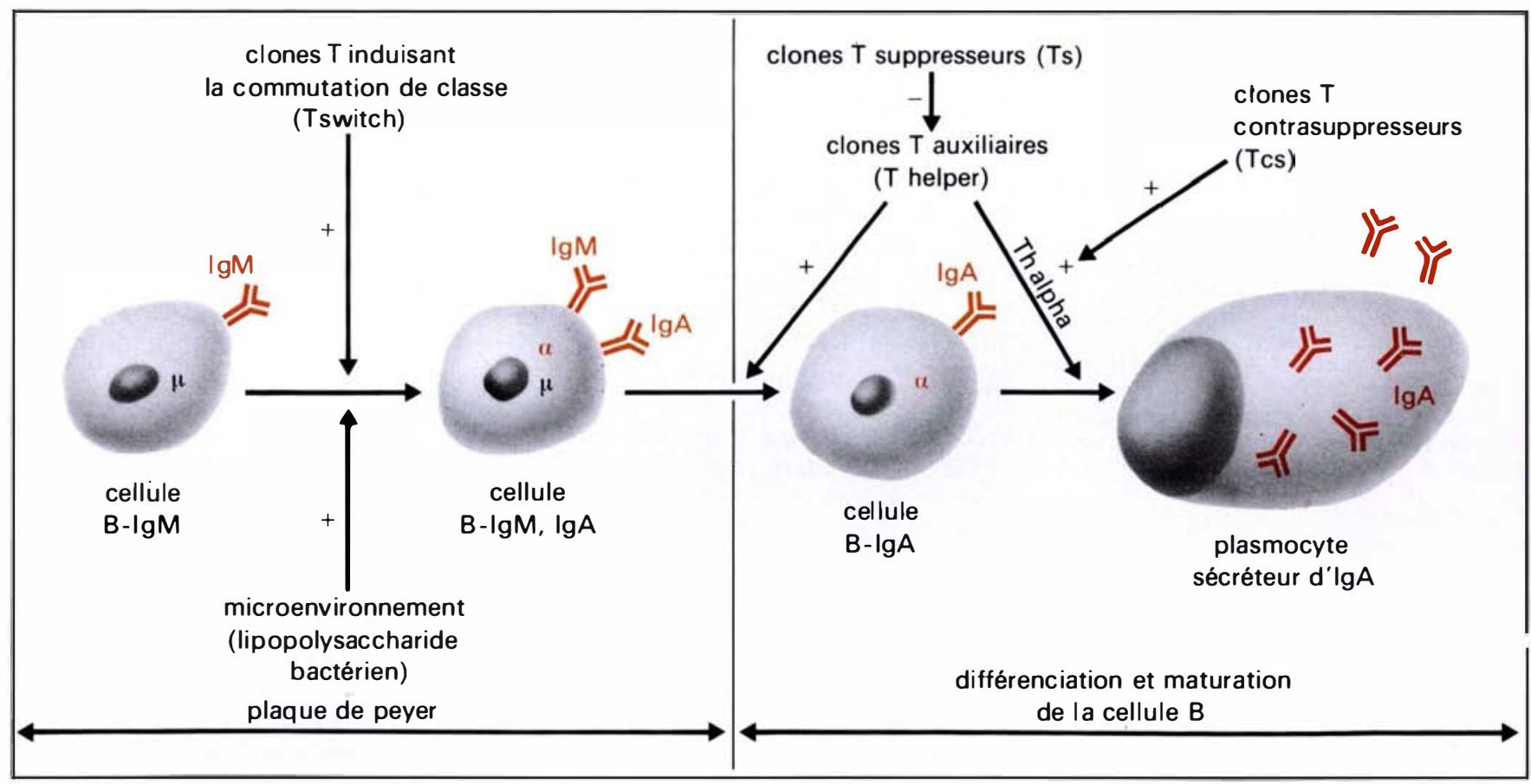

Figure 3. Rôle du micro-environnement et des sous-populations $T$ sur la différenciation et la prolifération des cellules $B$ du tissu lymphoïde associé au tractus digestif. 


\section{RĖFĖRENCES}

19. McGhce JR, Michalek SM, Kiyono H, et al. Mucosal immunoregulation: Finvironmental lipopolysaccharide and galt $\mathrm{T}$ lymphocytes regulatc the IgA rcsponse. Microbiol Immunol 1984; 28 : $261-80$.

20. Green DR, Saint-Martin S. Suppression and contrasuppression in the regulation of the gut associated immune responses. Ann NY Acad Sci 1983; 409: $284-90$

2I. Pierce NF, Gowans JL. Cellular kinetics of the intestinal immune response to cholera toxoid in rats. 7 Exp Med 1975 ; 142 : $1550-63$

22. Gow ans JI. Knight EJ. The route of recirculation of lymphoxytes in the rat. Proc $R$ Soc Lond [Biol] 1964; 159: 257-82.

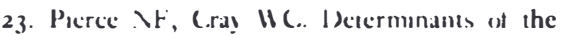
localization magnitude and duration of a specific mucosal IgA plasma cell response in enterically immunized rats. 7 Immunol I 982; 1 28: 13 I I-5.

24. Guy-Grand 1), Griscelli (, Vassali P. The gut associated lymphoid system: nature and properties of the large dividing cells. liur 7 Immunol $1974 ; 4: 435-43$.

25. Rambaud JC, Jian R, Galian A, Seligmann M. Maladies des chaines alpha. Presse Med 1985; I4: $155 \mathrm{I}-6$.

26. Mestecky J, McGhce J, Arnold RR, Michalek SM, Prince SJ, Babb JI. Selective induction of an immune response in human external secretions by ingestion of bacterial antigen. J Clin Invest 1978; 61: $73^{1-7}$

27. Kleinman RF, Walker WA. The enteromammary immune system. An important new concept in breast milk host defense. Dig Dis Sici 1979; 24: $876-82$.

28. Montgomery PC, Lemaitre-Coelho IM, Vacrman JP. A common mucosal immune system. Antibody expression in secretions following gastrointestinal stimulation. Immunol Commun I 980; 9: 705-I3

29. Rudzik R, (.lancy RI, Percy I)YF, D)ay RP, Bienenstock J. Repopulation with IgA containing cells of bronchial and intestinal lamina propria after transfer of homologous Pcycr's patch and bronchial lymphocytes. 7 Immunol 1975; II : I 599-I 604

3o. Weisz-Carrington P, Roux ME, McWilliams M, Philips-Quagliata JM, Lamm ME, Hormonal induction of the secretory immune system in the mammary gland. Proc Natl Acad Sci USA 1978; 75: $2928-32$.

31. Bienenstock J. The physiology of the local immune response. In: Asquith $\mathrm{P}$, ed. Immunolog) of the gastrointestinal tract. Fdinburgh: (hurchill Livingstone, 1979: 4.

32. Delacroix DL, Dive C, Rambaud JC, Vaerman JP. IgA subclasses in various secretions and in serum. Immunology' 1982; $47: 383-5$.

33. Owen RL. Sequential uptake of horseradish peroxidase by lymphoid follicle epithelium of Peyer's patches in the normal unobstructed mouse intestine: an ultra-structural study. Gastroenterolog)' 1977; 72: 440-51.
(Ts) spécifiques des isotypes IgM et IgG et migrant vers la rate, alors que la cellule $\mathrm{Th}$ alpha resterait cantonnée aux plaques de Peyer, ou s'y trouverait déréprimée par une autre cellule $T$ engagée dans une boucle contrasuppressive [20] ( $\mathrm{fi}$ gure 3). Une anomalie de cette boucle, ou l'existence d'une activité Ts IgA spécifique, pourrait expliquer le déficit sélectif en $\operatorname{Ig} \mathrm{A}$ qui frappe un sujet sur 700 environ. L'induction d'une tolérance systémique par présentation orale de l'antigène a fait naître l'espoir d'une approche nouvelle du traitement de l'allergie, mais ce phénomène ne s'observe malheureusement qu'en cas de primo-immunisation [21-23].

\section{Circuit des cellules Iymphoïdes B}

Nées dans les plaques de Peyer, les grandes cellules blastiques synthétisant l'IgA migrent par voie lymphatique vers les ganglions mésentériques pour lesquels elles ont une af finité particulière, peut-être liée à la présence, sur les cellules endothéliales, de récepteurs spécifiques [16]. Dans les ganglions, l'expansion clonale se poursuit, en même temps que se produit un processus de maturation en "plasmoblastes", traduit par l'apparition d'IgA intracytoplasmique [24]. C'est alors qu'intervient un phénomène décrit en 1964 [22], mais dont le mécanisme intime reste encore $\mathrm{mal}$ élucidé : réinjectés dans le sang d'un receveur syngénique, les "plasmoblastes" isolés de la lymphe du canal thoracique du rat migrent préférentiellement vers la lamina propria intestinale. La même affinité a été ensuite retrouvée pour les cellules blastiques des plaques de Peyer et des ganglions mésentériques, mais non pour celles isolées de la rate ou des ganglions périphériques [24]. Ce retour préférentiel à l'organe d'origine, qui a reçu la dénomination intraduisible de homing, se produit 24 à 48 heures après l'entrée des cellules blastiques dans la circulation générale. Extravasés dans la lamina propria intestinale, les "plasmoblastes " y achèvent leur différenciation en plasmocytes. Ces cellules, qui ne recirculent pas et dont la durée de vie est de 5 à 6 jours, synthétisent les anticorps sécrétés dans la lumière intestinale sous la forme d'IgAs et spécifiques de l'antigène présenté aux lymphocytes des plaques de Peyer.

Le rôle de cet antigène dans le homing des cellules immuno-compétentes spécifiques dont il a déterminé la production et la migration, était controversé, de même que l'existence d'une mémoire immunologique après immunisation entérale. D'une part, des " plasmoblastes" provenant d'adultes syngéniques étaient capables de coloniser l'intestin grêle de souriceaux nouveaux-nés ou axéniques, et même un intestin fœtal greffé sous la capsule rénale [5]. D'autre part, et à l'inverse, l'administration dans le côlon distal d'enfants colostomisés d'un vaccin poliomyélitique, induisait une réponse IgAs purement locale [10]. Chez le rat primo-immunisé par voie intrapéritonéale contre la toxine cholérique, la densité des cellules synthétisant les anticorps spécifiques de classe $\operatorname{Ig}$ A était maximale à l'endroit de l'intestin où avait été pratiqué le rappel antigénique [21]. Ces contradictions apparentes furent résolues grâce à un modèle ingénieux d'anses intestinales isolées de Thiry-Vella chez le rat [23]. Ce modèle permit de démontrer la coexistence d'un homing indépendant de l'antigène, précoce et de courte durée, suivi d'une colonisation durable de la seule anse soumise à la réadministration de l'antigène après primo-immunisation intra-péritonéale. Ce homing dépendant de l'antigène était dû à l'expansion clonale de petits lymphocytes mémoire, soit, pour $25 \%$ d'entre eux, immédiatement disponibles (cellules "sessiles" d'origine locale ou systémique), soit, pour $75 \%$ d'entre eux, originaires des plaques de Peyer et subissant le cycle décrit plus haut. I a proximité de la restimulation par rapport au site de primo-immunisation, ainsi que la durée de l'exposition à l'antigène augmentaient l'intensité et la durée de la réponse secondaire. De plus, le homing des cellules issues des formations lymphoïdes agrégées semble avoir, dans certains modèles 
expérimentaux, une certaine spécificité d'organe. Bien que le rôle de l'antigène dans le homing semble clairement établi, le mécanisme intime de celui-ci reste tout à fait obscur. L'administration d'anticorps bloquants anti-CS et anti-Ig A a permis d'éliminer le rôle de ces facteurs. L'existence, sur les cellules endothéliales des vaisseaux sanguins intestinaux, de récepteurs spécifiques des cellules lymphoïdes ayant transité par les plaques de Peyer a été postulée, mais non prouvée [16]. Quel qu'en soit le mécanisme intime, les modalités du homing rendent très bien compte de la topographie de l'infiltrat cellulaire de la maladie des chaînes alpha : intéressant le plus souvent la totalité de la longueur de l'intestin grêle, il peut s'étendre à l'estomac et au recto-côlon, et quelquefois se cantonner à ces deux organes [25].

\section{Tissu lymphoïde associé aux muqueuses}

Bien qu'il ait été le mieux étudié au niveau du tube digestif, le système IgA sécrétoire n'est pas limité à celui-ci, mais s'étend à toutes les muqueuses en relation avec le milieu extérieur (tableau II). De plus, ses diverses composantes ont des relations privilégiées qui ont donné naissance au concept de tissu lymphoïde associé aux muqueuses ou MALT [5]. Les analogies de structure de ce tissu d'un organe à l'autre sont particulièrement nettes entre les bronches et l'intestin grêle : même peuplement diffus de la lamina propria muqueuse par des plasmocytes synthétisant principalement de l'IgA dimérique; formations lymphoides agrégées similaires aux plaques de Peyer, comportant notamment des cellules épithéliales de surface spécialisées dans la pinocytose et le transport des antigènes, ainsi que des macrophages à corps tingibles semblables aux cellules dendritiques des plaques de Peyer. L'apparition d'anticorps spécifiques de type IgAs dans les sécrétions nasales après administration orale de vaccin poliomyélitique, ou dans les larmes et la salive, mais non dans le sang, après ingestion de Streptococcus mutans, apportait les premières preuves de l'unicité de l'im- munité humorale des muqueuses [26]. L'existence d'un axe entéromammaire était également démontré chez la lapine, puis chez la femme allaitante, chez laquelle apparaissaient dans le lait des anticorps spécifiques après infection intestinale à Salmonella typhi ou E. Coli [27].

La synthèse locale, par les plasmocytes du chorion, des anticorps ainsi produits était démontrée au niveau des bronches, de la parotide et du sein, après immunisation par voie orale par la ferritine, ainsi qu'au niveau de l'intestin grêle, après immunisation par voie bronchique [28]. Les expériences de transfert de cellules lymphoïdes isolées des plaques de Peyer et des formations agrégées des bronches à des animaux irradiés léthalement, montraient la possiblité d'un repeuplement croisé de la lamina propria des muqueuses intestinale et bronchique [29]. De même, était mise en évidence la capacité des cellules blastiques originaires du GALT de coloniser la glande mammaire et de s'y différencier en plasmocytes producteurs d'Ig A, sous l'influence des ostroprogestatifs, de la prolactine et de la cortisone, les androgènes exerçant un effet inhibiteur [30]. La participation de la muqueuse du col utérin à ce flux migratoire hormono-induit a été démontrée, mais la nature du ou des facteurs responsables de l'extravasation des cellules blastiques originaires du GALT dans les organes génitaux, reste inconnue. Le rôle de cet axe entéro-génital dans la protection passive du tube digestif du nouveau-né, lors de l'accouchement et pendant les premières semaines de la vie, est facile à concevoir. La finalité des interconnexions entre muqueuses digestives, respiratoires, lacrimales et urinaires est plus difficile à saisir, mais la possibilité pour un stimulus antigénique localisé d'induire, de façon plus ou moins dissociée de la réponse systémique, une réplique immunitaire étendue à l'ensemble des organes en contact avec le milieu extérieur, semble pouvoir constituer un moyen de défense particulièrement bien adapté à la pluralité des voies d'invasion que peuvent emprunter certains agents agresseurs

\section{Summary}

The first data on the gutassociated lymphoid tissue appeared early in this century. However, much of the knowledge on the IgA secretory system was obtained after the description of this new immunoglobulin class in 1959. Secretory $\operatorname{Ig} A$ is synthetized by the mucosal plasma cells as a dimer of $\operatorname{Ig} A$ molecules linked by the J chain. This polymeric $\operatorname{Ig} \mathrm{A}$ is transported through the enterocyte by its binding to the secretory component, which acts as a sacrificial receptor at the basolateral membrane of the cell. The main mechanism of action of secretory $\operatorname{Ig} \mathrm{A}$ is the coating of intraluminal antigens, which is responsible for its antibacterial, antitoxin, antiviral, antiparasitic and antidietary protein activities. Peyer's patches play a major role in antigen presentation to bone marrow-derived B lymphocytes. Clonal expansion and switch to A isotype are governed by several $T$ cell subpopulations and probably by living microorganisms of the commensal distal gut flora, which also trigger the development of the $\operatorname{Ig} A$ secretory system af ter birth. Further clonal expansion and carly differenciation into "plasmablasts" occur in mesenteric lymph nodes. The plasmablasts enter the systemic blood and selectively home not only to small intestinal lamina propria, but also to all mucosae in contact with the external milieu. Both antigen-independent and antigen-dependent factors govern this homing. Among them, sexual hormones and cortisol are responsible for the existence of an entero-sexual axis, which protects the newborn during delivery and first months of life.

\footnotetext{
TIRÉS A PART

J. C. Rambaud : Hôpital Saint-I.azare, r07, rue du Faubourg Saint-1)enis, 750 ro Paris.
} 